\title{
A COUNTABLE CONNECTED URYSOHN SPACE CONTAINING A DISPERSION POINT
}

\author{
V. KANNAN
}

\begin{abstract}
Answering a question of Martin, Roy gave the first example of a countable connected Urysohn space with a dispersion point. Here we give a much simpler example of such a space.
\end{abstract}

A Urysohn space is a topological space in which any two distinct points can be separated by disjoint closed neighbourhoods. A dispersion point in a connected space $X$ is a point $x$ such that $X \backslash\{x\}$ is totally disconnected. In [3], J. Martin asks: Does there exist a countable connected Urysohn space with a dispersion point? The first such example was given by Prabir Roy [5]. We give here a simpler example of such a space.

Let $R$ be the real line with the usual topology, and let $K$ be its one-pointcompactification. Let us denote the new point of $K$ by $\infty$. Take a copy of the set $Q$ of all rational numbers and keep it disjoint with $K$. For each $q$ in $Q$, let $q^{1}$ denote the same number considered as an element of $K$. On the disjoint union of $Q$ and $K$, define a topology as follows:

We take the usual topology on $K$ and declare this set to be open. An $\varepsilon$-neighbourhood (for any real number $\varepsilon>0$ ) of a point $q$ in $Q$ is a union of three sets:

(1) $\{q\}$;

(2) the usual $\varepsilon$-neighbourhood of $q^{1}-\sqrt{ } 2$ in $K$; and

(3) the usual $\varepsilon$-neighbourhood of $q^{1}+\sqrt{2}$ in $K$.

Declare these $\varepsilon$-neighbourhoods for all $\varepsilon>0$ to form a base at $q$ in $Q$. Now delete all the irrational points in $K$. Let $X$ be the subspace thus obtained.

It can be proved by straightforward methods that

(a) any open-and-closed set containing $\infty$ must be the whole $X$, and

(b) any two points of $X \backslash\{\infty\}$ can be separated by disjoint sets which are open-and-closed in $X \backslash\{\infty\}$.

These facts imply that $\infty$ is a dispersion point of $X$. The verification that $X$ is Urysohn is also straightforward.

Received by the editors July $16,1971$.

AMS 1970 subject classifications. Primary 54D05; Secondary 54G99.

Key words and phrases. Countable connected space. 
Remarks. (1) R. H. Bing [1] has given a simple example of a countable connected Hausdorff space $B$. It can be seen that in our example, $X \backslash\{\infty\}$ is homeomorphic to the subspace $\{(x, y) \in B \mid y=0$ or $y=1\}$ of $B$.

(2) Subsequently, the author has been able to prove the existence of $2^{c}$ mutually nonhomeomorphic countable connected Urysohn spaces with dispersion points. The proof of this result will be included in [2].

(3) It can be seen that the space $X$ in the above example is quasimetrisable. Thus it supplements a result of [4].

The author thanks the referee for some of his suggestions.

\section{REFERENCES}

1. R. H. Bing, A connected countable Hausdorff space, Proc. Amer. Math. Soc. 4 (1953), 474. MR 15, 729.

2. V. Kannan and M. Rajagopalan, Countable topological spaces, Technical Publications of the Dept. of Mathematics, Madurai University (to appear).

3. J. Martin, A countable Hausdorff space with a dispersion point, Duke Math. J. 33 (1966), 165-167. MR 33 \#699.

4. G. G. Miller, Countable connected spaces, Proc. Amer. Math. Soc. 26 (1970), 355-360.

5. Prabir Roy, A countable connected Urysohn space with a dispersion point, Duke Math. J. 33 (1966), 331-333. MR 33 \#4887.

Department of Mathematical Science, Madurai University, Madurai-2, India 